\title{
Computational Soundness of Coinductive Symbolic Security under Active Attacks
}

\author{
Mohammad Hajiabadi and Bruce M. Kapron \\ Dept. of Computer Science, University of Victoria, Victoria, CANADA V8W 3P6 \\ \{mhaji, bmkapron\}@cs.uvic.ca
}

\begin{abstract}
In Eurocrypt 2010, Miccinacio initiated an investigation of cryptographically sound, symbolic security analysis with respect to coinductive adversarial knowledge, and showed that under an adversarially passive model, certain security criteria may be given a computationally sound symbolic characterization, without the assumption of key acyclic$i t y$. Left open in his work was the fundamental question of "the viability of extending the coinductive approach to prove computational soundness results in the presence of active adversaries." In this paper we make some initial steps toward this goal with respect to an extension of a tracebased security model (Micciancio and Warinschi, TCC 2004) including asymmetric and symmetric encryption; in particular we prove that a random computational trace can be soundly abstracted by a coinductive symbolic trace with overwhelming probability, provided that both the underlying encryption schemes provide IND-CCA2 security (plus ciphertext integrity for the symmetric scheme), and that the diameter of the underlying coinductively-hidden subgraph is constant in every symbolic trace. This result holds even if the protocol allows arbitrarily nested applications of symmetric/asymmetric encryption, unrestricted transmission of symmetric keys, and adversaries who adaptively corrupt users, along with other forms of active attack.

As part of our proof, we formulate a game-based definition of encryption security allowing adaptive corruptions of keys and certain forms of adaptive key-dependent plaintext attack, along with other common forms of CCA2 attack. We prove that (with assumptions similar to above) security under this game is implied by IND-CCA2 security. This also characterizes a provably benign form of cyclic encryption implied by standard security definitions, which may be of independent interest.
\end{abstract}

Keywords: Computational soundness, adaptive corruptions, coinduction, circular security, trace-based protocol security, active adversaries.

\section{Introduction}

Provable security, since its introduction in the early 1980s, has provided a rigorous foundation for the security analysis of cryptographic schemes. Typically, proving a cryptographic construction meets a given security goal within the provable security framework requires: (1) formally defining the security goal in

A. Sahai (Ed.): TCC 2013, LNCS 7785, pp. 539-558, 2013.

(C) International Association for Cryptologic Research 2013 
terms of what comprises a violation of the goal and what is assumed about the computational power of the adversary, and (2) giving a feasible method which transforms any attack against the construction to an attack against one of its underlying primitives 1213. This methodology provides strong security assurances against resource-bounded attackers, which is a fairly realistic assumption in real-world applications. However, doing computational security analysis, even for small-sized protocols, can be a gruelingly tedious task, and normally a small change in the protocol necessitates a new security proof. On the other hand, formal (logic-based) methods 24,15] greatly simplify security analysis using idealized abstractions of cryptographic primitives and limiting adversarial computation, even allowing for automated verification. While formal methods may help designers identify subtle flaws in their schemes, they do not necessarily provide guarantees of computational security. At the very least, a formally verified scheme may be computationally insecure if realized under "insufficiently strong" primitives (e.g. using malleable encryption in the case of active attacks). Motivated by the mismatch between these two approaches, a large body of work, starting from [1, attempts to give computational justifications for formal security proofs, in the form of computational soundness theorems. Generally speaking, a formal system for security proofs is computationally sound if whenever a scheme is proved secure in the system, it is guaranteed to also be secure in an appropriate computational security framework.

Background. Standard notions of secure encryption 2638 ensure privacy of plaintexts chosen independently from the underlying secret key(s). It has long been known that a key encrypted under itself may no longer remain secret, and recent results 202 show that indeed for all $k \geq 1, k$-circular security is not implied by standard security. Moreover, currently known techniques for standard security fall short when trying to prove non-trivial security statements against more adaptive adversaries. As an example, assume in the standard multiple-key-based indistinguishability game [9] over keys $c k_{1}, \ldots, c k_{n}$, the adversary is additionally allowed to obtain the (nested) encryption of any $c k_{i}$ under $\left\{c k_{1}, \ldots, c k_{i-1}\right\}$, giving rise to an acyclic encryption ordering between keys. One can use a standard hybrid argument to show that security in this setting is no stronger than standard security. However, this simple hybrid argument fails in the case that the (acyclic) encryption ordering is a priori unknown and formed adaptively by the adversary. (The naive approach of guessing the underlying ordering also trivially yields an exponential reduction factor.) In contrast, conventional Dolev-Yao style security analysis models adversarial knowledge inductively in an all-or-nothing fashion (i.e. the adversary either knows a secret piece of data, or it does not have any information about it). As a result, adversarial power is limited, essentially treating uniformly all symbolic ciphertexts whose encryption keys are underivable under so-called Dolev-Yao deduction rules. Consequently, Dolev-Yao models typically assume no difference between two symbolic encryptions $\{k\}_{k}$ and $\left\{k_{1}\right\}_{k}$. Also, the "adaptive problem" described above seems to not be a challenge within these models. For these reasons, most existing soundness results are restricted in their assumptions, which include excluding key cycles 
altogether in the case of passive adversaries 128, posing certain encryption orderings in the case of passive-but-adaptive adversaries [33, and disallowing symmetric encryption in the case of active adversaries [346 19].

As a resolution to the problems created by key cycles, Micciancio 32 proposes a coinductive method for modeling symbolic security, and obtains computational soundness in the setting of message indistinguishability for passive adversaries, while allowing key cycles and assuming only semantic security for the underlying encryption function. Coinductive symbolic security corresponds to a greatestfixedpoint-based definition of adversarial knowledge, as opposed to the leastfixedpoint-based definition adopted by conventional inductive methods. From a cryptographic perspective, 32 implicitly characterizes a provably benign form of circular encryption, in particular the equivalence of standard security to secure encryption under a variant of the multiple-key-based game described above in which the adversary may obtain the (single or nested) encryption of any $c k_{i}$ under arbitrary keys, provided at least one of them is in $\left\{c k_{1}, \ldots, c k_{i-1}\right\}$, resulting in a (possibly) cyclic encryption ordering. To obtain soundness, 32 shows that for an a priori known sequence of exchanged symbolic messages (which is the case in the passive setting), one may order all coinductively irrecoverable keys from this sequence as $k_{1}, \ldots, k_{m}$, such that each occurrence of $k_{i}$ is encrypted under at least one of $\left\{k_{1}, \ldots, k_{i-1}\right\}$.

Our Results. In this paper we investigate the question left open in [32; namely, whether a coinductive approach provides similar soundness guarantees when applied in the setting of active adversaries. We consider a symbolic/computational trace-based execution model 34, including asymmetric and symmetric encryption. In contrast to previous work, we allow symmetric keys to be freely included in protocol messages, symmetric and asymmetric encryptions to be arbitrarily nested, and adversaries to adaptively corrupt users, along with other forms of active attack. We first pose the following central question: to what extent can any encryption scheme with standard security withstand stronger types of attack including adaptive corruptions of keys and key-dependent/circular encryption? To formalize this, consider the following game over symmetric/asymmetric encryption schemes $\mathcal{E}^{s}=\left(G^{s}, E^{s}, D^{s}\right), \mathcal{E}^{a}=\left(G^{a}, E^{a}, D^{a}\right),\left\{c k_{i}\right\}_{1 \leq i \leq n} \leftarrow G^{s}\left(1^{\eta}\right)$, and $\left\{\left(p k_{i}, s k_{i}\right)\right\}_{1 \leq i \leq n} \leftarrow G^{a}\left(1^{\eta}\right)$, in which the adversary is allowed to adaptively corrupt keys (symmetric and asymmetric), obtain decryptions of permissible ciphertexts, and issue key-dependent encryption queries of the form $E^{s}\left(f\left(c k_{1}, \ldots, c k_{n}\right), c k_{j}\right)$ or $E^{a}\left(f\left(c k_{1}, \ldots, c k_{n}\right), p k_{j}\right)$, where $f$ is any arbitrary composition of constant, pairing, projection $\left(P_{i}\left(c k_{1}, \ldots, c k_{n}\right)=c k_{i}\right)$, and encryption $\left(E_{p k_{i}}^{a}(\cdot), E_{c k_{i}}^{s}(\cdot)\right)$ functions. We remark asymmetric decryption keys may not be used to form key-dependent messages, reflecting our assumption that such keys are not sent as plaintexts in protocol messages 1 . This function family allows one to describe encryption queries symbolically (e.g. $E^{s}\left(E^{s}\left(c k_{1}, c k_{2}\right), c k_{1}\right)$ is denoted $\left\{\left\{k_{1}\right\}_{k_{2}}\right\}_{k_{1}}$ ), and hence symbolically keep track of adversarial knowledge. Now we ask: if $\mathcal{E}^{a}$ and $\mathcal{E}^{s}$ provide IND-CCA2 security only, can we prove,

${ }^{1}$ Relaxing this requirement does not add to the technical difficulty of the proofs. We assumed this requirement as it seems to be the case for most protocols in practice. 
at the end of the game, certain keys still maintain computational secrecy, in the sense they can securely be used in an encryption-based indistinguishability game2? Several negative results 2022 show certain key cycles may compromise the secrecy of their component keys, but on the positive side this problem (in a generic sense involving circular encryption) has not been considered much. Motivating the discussion, the results of 32 in the context of the above game (but where only symmetric encryption is used,) imply if all queries are made at once (i.e. nonadaptively), then any $c k_{i}$, whose symbolic key $k_{i}$ remains coinductively irrecoverable (irrecoverable for short), even if used in key cycles, maintains computational secrecy. Along these lines, we call $\left(\mathcal{E}^{a}, \mathcal{E}^{s}\right) C I$ secure if after the adaptive execution of the above game all keys whose symbolic keys remain irrecoverable maintain computational secrecy. We also consider ACI security, an extension of CI security which adds ciphertext integrity and obtain the following

Theorem (informal). If $\left(\mathcal{E}^{a}, \mathcal{E}^{s}\right)$ is ACI secure, it provides soundness for coinductive traces.

Next we ask if CI security may be based on IND-CCA2 security. Note that the CI attack model is ostensibly much stronger than the CCA2 one, allowing a CI adversary to adaptively corrupt keys and obtain circularly-encrypted ciphertexts. A naive reduction attempt would be to a priori guess all keys which remain irrecoverable during the game, together with their underlying encryption ordering, and then use a hybrid argument in the style of 32 to do the reduction. Such an idea clearly yields an infeasible reduction factor. Instead, we prove that if the diameter of the coinductively-hidden subgraph of the resulting key graph is constant, then CI security is implied by IND-CCA2 security. (It will soon be informally described why our reduction is dependent on this parameter.) Here, the key graph is the (random) multigraph $G_{k}$ which has a node for every key in the game, and an edge $v_{i} \rightarrow v_{j}$ if $v_{i}$ 's associated key encrypts $v_{j}$ 's in an encryption query (e.g. the encryption query $\left\{\left\{k_{1}\right\}_{k_{2}}\right\}_{k_{1}}$ creates one self-loop and one normal edge,) and by "coinductively hidden subgraph" we mean the induced subgraph of $G_{k}$ on irrecoverable nodes (nodes whose associated keys remain irrecoverable). We remark that as long as the above condition holds, the adversary may corrupt any number of keys, and create arbitrary key cycles and arbitrarily-long paths in the whole key graph.

Theorem (informal). If $\mathcal{E}^{a}$ and $\mathcal{E}^{s}$ are both IND-CCA2 secure, then for every adversary $\mathcal{A}$ where the diameter of the coinductively hidden subgraph of $G_{k}(\mathcal{A})$ is constant (i.e. independent of the security parameter), $\mathcal{A}$ has a negligible advantage in the CI game for $\left(\mathcal{E}^{a}, \mathcal{E}^{s}\right)$. Moreover, if $\mathcal{E}^{s}$ is also INT-CTXT secure, $\mathcal{A}$ has a negligible advantage in the ACI game.

The starting point of our proof is [36]'s positive results on security against adaptive corruptions (in an authenticated channel setting), showing that security

${ }^{2}$ Our definition of computational secrecy is close to the idea of key usability, developed in 23, for defining alternate, composition-amenable security criteria for key-exchange protocols. 
in a setting over $\mathcal{E}^{s}$ and $\left\{c k_{i}\right\}_{1 \leq i \leq n} \leftarrow G^{s}\left(1^{\eta}\right)$, in which $\mathcal{A}$ may adaptively corrupt keys, and obtain single encryptions $E^{s}\left(c k_{i}, c k_{j}\right)$, for $1 \leq i, j \leq n$, subject to key acyclicity, is obtained via a reduction to the semantic security of $\mathcal{E}^{s}$, with a factor of $O\left(n^{l}\right)$ where $l$ is the diameter of the resulting key graph. Although the results of [36] seem to extend, by its mere developed techniques, to an authenticated setting with nested encryptions, they crucially rely on acyclicity and break down if this latter is relaxed. Allowing cyclic nested encryptions, irrecoverable nodes may have self-loops or oppositely-directed edges between themselves (encryption queries $\left\{\left\{k_{1}\right\}_{k_{2}}\right\}_{k_{3}}$ and $\left\{k_{2}\right\}_{k_{1}}$ create such edges, while $k_{1}, k_{2}$ remain irrecoverable), and we still need to prove their computational secrecy. Central to our proof is a new notion of coinductive continuability, which for every irrecoverable node characterizes a special set of paths ending in that node, satisfying a property which enables a path-based reduction proof in the style of [36]. (Our reduction is based on guessing random coinductiely continuable paths with certain properties, making it depend on the diameter.) Also, allowing both nested encryptions and decryption queries creates a new complication; namely, to simulate a CI adversary $\mathcal{A}^{C I}$ by a CCA2 adversary $\mathcal{A}^{c c a}$, nested encryptions may make

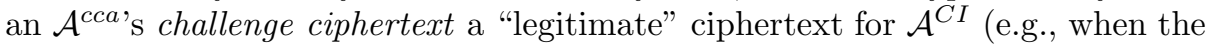
ciphertext corresponding to $\left\{k_{1}\right\}_{k_{2}}$ in $\left\{\left\{k_{1}\right\}_{k_{2}}\right\}_{k_{3}}$ is created under $\mathcal{A}^{c c a}$ 's left-orright oracle and $k_{3}$ remains irrecoverable), and if $\mathcal{A}^{C I}$ makes such a decryption query, our simulation fails. A large part of our proof, thus, involves showing $\mathcal{A}^{C I}$ may produce such ciphertexts only with negligible probability. Such a complication does not arise if one only deals with single encryptions, and in fact, the results of [36] immediately extend if decryption queries are also allowed.

Applications. Our reduction result implies for a protocol $\Pi$ (which may contain symmetric keys and nonces as atomic messages) and a trace-expressible security property $\mathcal{P}$ (here, loosely speaking, by a trace we mean a sequence of states created during an execution of a protocol as a result of adversarial/honest-parties' actions. Formal definitions are given in Section 3), if the following two symbolic assertions hold, then the (CCA2, CCA2+CTXT)-based implementation of $\Pi$ provably achieves $\mathcal{P}$ (in an insecure channel setting) with strong security guarantees against adaptive corruptions: (a) No symbolic coinductive adversary may create a trace containing an arbitrarily-long encryption chain (in the sense described above), and (b) $\Pi$ is coinductively secure; namely, no coinductive symbolic adversary may produce a trace not satisfying the underlying symbolic property. We observe that all protocols in the Clark-Jacob library 21, in which the only primitives used are asymmetric/symmetric encryption, satisfy our soundness restriction (item (a) above), making it applicable to them. A number of these protocols are asymmetric encryption-based, and analyzable under previous soundness theorems (e.g. 346]). Using our techniques, we show that [27] the Wide-Mouthed Frog authentication protocol, which is not analyzable under the cryptographic library of [4] due to the classic commitment problem prevalent in simulation-based approaches, satisfies our soundness restriction. This advocates for the use of coinduction as a strong tool in yielding provably-sound security proofs, while circumventing issues involved with using induction-based methods. 
Why Not KDM Security? It may be asked why we bother to investigate soundness of coinductive methods, when there are constructions in the standard model for secure encryption under key-dependent messaging [14]16. We note that security against adaptive corruptions is a necessary requirement for any encryption scheme used in a protocol which is run in an environment with adversarially adaptive corruptions. In such situations, once a key is corrupted, the security of the protocol will depend on the preservation of secrecy for keys which are not trivially corrupted. Even in the idealized static corruption model, a key may dynamically be revealed by the exploitation of potential weaknesses of a protocol (e.g., consider a situation where the adversary gets to alter a communicated message by replacing an "honest" key with his own key, making an honest party then encrypt a secret key under the adversarial key.) To the best of our knowledge, there are no provable constructions of KDM-secure encryption in the standard model which also provide security against adaptive corruptions. Backes et al. [5] consider a limited case in which security is defined only in a left-or-right indistinguishability sense, not addressing the above problem. In subsequent work, [3] considers the problem in its full generality as described above, but their construction is in the random-oracle model. Moreover, they do not consider the question of whether generic constructions from KDM-secure encryption schemes exist (in the standard model) which also provide security against adaptive corruptions.

Related Work. Obtaining sound abstract security proofs for protocols involving symmetric encryption has also been considered following the ideal/real simulation paradigms of [1737. 4] shows that secure realization of ideal symmetric encryption (in the sense of reactive simulatability) is possible in their cryptographic library [6] if the commitment problem does not occur (i.e. any honest party's key, after it is used for encryption, never becomes "known" to the adversary), and the used-order property is satisfied. (i.e. Deployed keys admit an a priori encryption ordering.) The authors of [30], by extending the framework of [19] to allow symmetric encryption, show if a key-exchange protocol satisfies their symbolic criteria and if the above conditions hold, the protocol securely realizes a key-exchange functionality in the sense of universal composability. We comment the commitment problem may intrinsically occur as a direct result of security formalizations; adaptive corruptions, for instance, trivially enable this possibility. Also, the requirement that "a session-key loss in a key-exchange protocol should not affect the secrecy of other session keys" is formalized by allowing the adversary to adaptively learn session keys, leading, possibly, to the commitment problem. Thus the aforementioned frameworks do not consider the above two attack scenarios. We remark the commitment problem was known long before in the setting of adaptively-secure multiparty computation, with initial solutions given in [18]. The results of [22] are aimed at indistinguishability-based security properties (e.g., secrecy requirements for key-exchange protocols), by showing that observational equivalence between two processes implies computational indistinguishability under standard cryptographic assumptions. Although 22] allows symmetric encryption, it imposes the same restrictions as 4430]. 
A very different approach which in principle supports reasoning about situations which include key-cyclic encryption and adaptive corruption for both symmetric and asymmetric encryption as well as other primitives is the use of what might be called general-purpose security logics. Here we include probabilistic process calculi [3135], logics which axiomatize computational indistinguishability 298] and first-order logics augmented with axioms characterizing specific security properties [7. The tradeoff involved in taking a more generic approach is the loss of structure in proofs, potentially undermining some of the benefits of the formal approach.

Basic Notation: For a review of the standard notions of encryption security, we refer to 1011 . If $D$ is a probability distribution, then $x \leftarrow D$ denotes choosing an element according to $D$, and if $S$ is a set, $x \leftarrow S$ denotes choosing an element uniformly at random from $S$. For a probability distribution $D$, sup $[D]$ denotes the support set of $D$, and we write $x \in D$ to mean $x \in \sup [D]$. We call a function negligible if it grows more slowly than the inverse of any polynomial function. For ease of notation, we use $\operatorname{negl}(\cdot)$ to refer to any negligible function.

\section{Preliminaries}

A Formal Language for Cryptographic Expressions. Expressions are built from four infinite sets of basic symbols - identifiers, ID, public-key symbols, $\mathcal{K}^{p u b}$, private-key symbols $\mathcal{K}^{\text {priv }}$, and nonces, $\mathcal{X}$ - using encryption, $\{\diamond\}_{\circ}$, and concatenation, $(\cdot, \cdot)$, operators for building compound messages. We further partition $\mathcal{K}^{\text {priv }}$ into asymmetric private keys, $\mathcal{K}^{\text {privasym }}$, and symmetric private keys $\mathcal{K}^{\text {privsym }}$. We fix a bijective key-inverse operation $(.)^{-1}: \mathcal{K}^{\text {pub }} \cup \mathcal{K}^{\text {privsym }} \rightarrow$ $\mathcal{K}^{\text {priv }}$, which induces the identity function on subdomain $\mathcal{K}^{\text {privsym }}$.

Whenever it is essential to distinguish between the adversary's and honest parties' basic symbols, we add a subscript $A$ or $H$ to basic symbols, and for every set $S$ defined above, we further define $S=S_{H} \cup S_{A}$ (e.g. $\mathcal{K}_{H}^{\text {priv }}$ and $\mathcal{K}_{A}^{\text {priv }}$ ). Moreover, whenever it is necessary to distinguish between symmetric and asymmetric private-key symbols, we add a superscript sym to symmetric ones. (e.g. we have $\left(k_{1}^{s y m}\right)^{-1}=k_{1}^{s y m}$.) The set of formal expressions, Exp, is:

$$
\begin{aligned}
& \text { Exp }::=\text { Plain } \mid \text { Cipher } \mid(\text { Exp, Exp }) \\
& \text { Plain ::=ID }|\mathcal{X}| \mathcal{K}^{\text {pub }} \mid \mathcal{K}^{\text {privsym }} \\
& \text { Cipher }::=\{\text { Plain }\}_{k \in \mathcal{K}^{p u b} \cup \mathcal{K}^{\text {privsym }}} \mid\{\text { Cipher }\}_{k \in \mathcal{K}^{\text {pub }} \cup \mathcal{K}^{\text {privsym }}}
\end{aligned}
$$

Coniductive Modeling of Adversarial Knowledge. We take a coinductive approach to modeling adversarial attacks. To model coinductive adversarial knowledge [32, we define a key-recovery function, $\mathcal{F}$, which specifies given $e \in \operatorname{Exp}$ and $T \subseteq \mathcal{K}_{H}^{\text {priv }}$, what keys can be deduced by "single-round" applications of Dolev-Yao rules. Defined naturally, $\mathcal{F}_{s}(T)=s \cap \mathcal{K}_{H}^{\text {priv }}$ for a basic symbol $s, \mathcal{F}_{\left(e_{1}, e_{2}\right)}(T)=\mathcal{F}_{e_{1}}(T) \cup \mathcal{F}_{e_{2}}(T)$, and $\mathcal{F}_{\{e\}_{k}}(T)=\mathcal{F}_{e}(T)$ if $k^{-1} \in T \cup \mathcal{K}_{A}^{\text {priv }}$ and $\mathcal{F}_{\{e\}_{k}}(T)=\emptyset$, otherwise. $T$ is a fixedpoint of $\mathcal{F}_{e}$ if $\mathcal{F}_{e}(T)=T$, and is the greatest (resp. least) fixedpoint if $T$ is the greatest (resp. least) solution of $\mathcal{F}_{e}(X)=X$ 
(according to $\subseteq$ ordering). Now $T$ is coinductively (resp. inductively) defined by $\mathcal{F}_{e}$ if $T$ is the greatest (resp. least) fixedpoint of $\mathcal{F}_{e}$. It is easy to see that $\mathcal{F}_{e}$ is a monotone function (i.e., $S_{1} \subseteq S_{2} \Rightarrow \mathcal{F}_{e}\left(S_{1}\right) \subseteq \mathcal{F}_{e}\left(S_{2}\right)$.

The Tarski-Knaster Theorem implies for every monotone function $F: \wp(D) \rightarrow$ $\wp(D)$, where $D$ is some set and $\wp(D)$ is its powerset, the least fixedpoint, fix $(F)$, and greatest fixedpoint, $F I X(F)$, of $F$ exist and are obtained as follows

$$
f i x(F)=\bigcap_{S: F(S) \subseteq S} S \quad(1) \quad F I X(F)=\bigcup_{S: S \subseteq F(S)} S
$$

Note that if $T \subseteq \mathcal{F}_{e}(T)$, then $\operatorname{cl}(T) \triangleq \cup_{i \geq 1} \mathcal{F}_{e}^{i}(T)$ is a fixedpoint, for which $T \subseteq \operatorname{cl}(T)$, where $\mathcal{F}_{e}^{i}(T)$ denotes $i$ successive applications of $\mathcal{F}_{e}$ on $T$. The latter follows from monotonicity of $\mathcal{F}_{e}$, and the former follows observing that $\mathcal{F}_{e}^{k}(T)=\mathcal{F}_{e}^{k+1}(T)$ for sufficiently large $k$ 's. (This is because the number of keys in $e$ is finite.) Thus the following equivalent formulations follow:

$$
\operatorname{fix}\left(\mathcal{F}_{e}\right)=\bigcap_{\mathcal{F}_{e}(S)=S} S=\bigcup_{i \geq 1} \mathcal{F}_{e}^{i}(\emptyset)
$$

$$
F I X\left(\mathcal{F}_{e}\right)=\bigcup_{S=\mathcal{F}_{e}(S)} S=\bigcap_{i \geq 1} \mathcal{F}_{e}^{i}\left(\mathcal{K}_{H}^{\text {priv }}\right)
$$

We show (4); the proof for (3) follows by a dual argument. The first equality for $F I X\left(\mathcal{F}_{e}\right)$ follows from (2) and the argument presented above. The second equality follows from the following three observations: (a) $\bigcap_{i>1} \mathcal{F}_{e}^{i}\left(\mathcal{K}_{H}^{\text {priv }}\right)$ is a fixedpoint of $\mathcal{F}_{e}$, (b) if $T$ is a fixedpoint of $\mathcal{F}_{e}$, then $T=\bigcap_{i \geq 1} \mathcal{F}_{e}^{i}(T)$, and (c) by monotonicity, $\bigcap_{i>1} \mathcal{F}_{e}^{i}(T) \subseteq \bigcap_{i>1} \mathcal{F}_{e}^{i}\left(\mathcal{K}_{H}^{\text {priv }}\right)$. Now the set of coinductively recoverable keys of $e$ is the set coinductively defined by $\mathcal{F}_{e}$. For example for $e=k^{-1},\left\{\left\{k_{1}^{s y m}\right\}_{k_{2}^{s y m}},\left\{k_{2}^{s y m}\right\}_{k_{1}^{s y m}}\right\}_{k}$, its coinductively recoverable keys are $\left\{k^{-1}, k_{1}^{s y m}, k_{2}^{s y m}\right\}$. (As a convention, we omit parentheses in expressions and write $e_{1}, e_{2}$ for $\left(e_{1}, e_{2}\right)$.) See 32 for more examples.

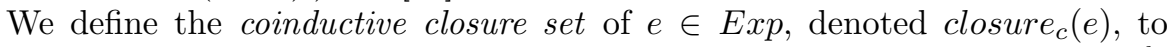
be the smallest set satisfying: (i) closure $_{c}(e)$ contains $e, F I X\left(\mathcal{F}_{e}\right), I D, \mathcal{K}^{p u b}$, and all the adversary's basic symbols, (ii) if $\left(e_{1}, e_{2}\right) \in$ closure $_{c}(e)$ then $e_{1}, e_{2} \in$ closure $_{c}(e)$, (iii) if $e^{\prime}$ and $e^{\prime \prime}$ are both in closure $_{c}(e)$, so is $\left(e^{\prime}, e^{\prime \prime}\right)$, (iv) if $\{m\}_{k} \in$ closure $_{c}(e)$ and $k^{-1} \in$ closure $_{c}(e)$ then $m \in \operatorname{closure}_{c}(e)$, and (v) if $m \in$ closure $_{c}(e)$ and $k \in$ closure $_{c}(e)$ then $\{m\}_{k} \in$ closure $_{c}(e)$. Although the above definition is a hybrid of inductive and coinductive definitions, an equivalent, (fully) coinductive definition is also possible; however, we adopt the above one as it is more natural. Now $e_{1}$ is coinductively recoverable from $e$ if $e_{1} \in$ closure $_{c}(e)$. Note, if $e_{1} \in$ closure $_{c}(e)$ but $k^{\text {sym }} \notin$ closure $_{c}(e)$, Rule (v) does not allow us to deduce $\left\{e_{1}\right\}_{k^{\text {sym }}} \in$ closure $_{c}(e)$. This models the idealized symbolic assumption that if the adversary does not know an honest party's symmetric key, he cannot produce a ciphertext which decrypts to a meaningful plaintext under that key. To support this assumption in our computational model, we will assume the symmetric encryption scheme provides ciphertext integrity.

We say $e^{\prime}$ is a subexp of (or occurs in) $e$, denoted $e^{\prime} \sqsubseteq e$, if $e=e^{\prime}$, or $e=\left(e_{1}, e_{2}\right)$ and $e^{\prime} \sqsubseteq e_{1}$ or $e^{\prime} \sqsubseteq e_{2}$, or $e=\left\{e_{1}\right\}_{k}$ and $e^{\prime} \sqsubseteq e_{1}$. We say $k_{1}$ encrypts $k_{2}^{-1}$ in 
$e$, denoted $k_{1} \rightarrow^{e} k_{2}^{-1}$, if for some $\left\{e_{1}\right\}_{k_{1}}$ which occurs in $e, k_{2}^{-1} \sqsubseteq e_{1}$. An expression is key cyclic if it contains a key cycle, that is a sequence $k_{0}, k_{1}, \ldots k_{i-1}$ such that $k_{j} \rightarrow k_{(j+1 \bmod i)}^{-1}$ for all $j \geq 0$, and is called key acyclic if it is not key cyclic. It is known the inductive and coinductive definitions coincide for keyacyclic expressions 32 . The converse of this, however, does not hold true; it is possible some keys occur in certain key cycles but remain coinductively irrecoverable (e.g. consider $\left\{\left\{k_{1}^{-1}\right\}_{k_{1}}\right\}_{k_{2}}$ ). In fact, we will prove it is exactly such keys that remain "secure" under concrete implementations.

Computational Interpretation of Cryptographic Expressions. Under a pair of symmetric/asymmetric schemes $\mathcal{E}_{p}=\left(\mathcal{E}_{\text {sym }}, \mathcal{E}_{a s y}\right)$ with parameters $\left(\eta_{\text {sym }}, \eta_{\text {asy }}\right)$, an invertible pairing function, and a mapping $\tau\left(\eta_{\text {sym }}, \eta_{\text {asy }}, \circ\right)$, which gives a concrete value to every basic symbol, every $e \in \operatorname{Exp}$ induces a natural probability distribution, denoted $\llbracket e \rrbracket_{\tau}^{\mathcal{E}_{p}}$, which we call the computational image of $e$ with respect to $\mathcal{E}_{p}$ and $\tau$. If $E \in \llbracket e \rrbracket_{\tau}^{\mathcal{E}_{p}}$ and $e_{1} \sqsubseteq e$, given $\tau$, one may define the underlying value of $e_{1}$ in $E$ in a natural way.

\section{Symbolic and Computational Trace-Based Protocol Security}

We will now introduce a protocol specification language and consider an extension of the model given in 34 for analyzing security protocols in the presence of active adversaries. For simplicity, we consider two-party protocols, and assume that each protocol runs in a constant number of rounds, and admits a symbolic specification. Under these assumptions, a protocol can be described as a sequence $\Pi=\left(M_{1}^{I}, M_{1}^{R}, M_{2}^{I}, M_{2}^{R}, \ldots M_{r}^{I}, M_{r}^{R}\right)$ of messages being sent alternately between two parties: initiator and responder. (Here having the responder send the last message is arbitrary.) We assume that each party has an associated longlived public key which the other party may use to encrypt messages, and whose matching private key is never sent as a plaintext. The parties, however, may generate fresh symmetric keys, send them (encrypted) to each other, and later on use exchanged keys to encrypt future messages. Messages that we use to specify protocols are built upon four disjoint sets $I d s=\{I, R\}$, nonces $=\left\{X_{1}, X_{2}, \ldots\right\}$, pubkeys $=\left\{K_{I}, K_{R}\right\}$, and symkeys $=\left\{K_{1}^{\text {sym }}, K_{2}^{\text {sym }}, \ldots\right\}$, using encryption and concatenation for building compound messages, where $K_{I}$ and $K_{R}$ denote the parties' respective public keys. We further require protocols be computationally executable; in particular, a party should be able to fully decrypt (all encrypted parts of) a message she receives. (Our results seem to easily extend by relaxing this restriction, allowing, e.g., ciphertext forwarding, which allows a party to forward a message without decrypting it.) To summarize our assumptions, we call $\Pi$ valid if: (1) for all $1 \leq i \leq r$ and $x \in\{I, R\} ; K_{I}^{-1}$ and $K_{R}^{-1}$ do not occur in $M_{i}^{x}$, and (2) for all $1 \leq i \leq r, x \in\{I, R\}$, and $y=\{I, R\}-\{x\}$; if $M_{i}^{x}$ has a subexp $\{M\}_{K}$, then $K$ is inductively recoverable from $\left(K_{y}, M_{1}^{x}, \ldots, M_{i}^{x}\right)$. (We will use a coinductive approach for modeling adversarial attacks, and this condition is solely meant to specify our class of protocols. In particular, since we 
require parties be able to fully decrypt their received messages, and their roles be computationally executable, such a condition seems necessary.)

So far we have only described the "syntax" of protocols; this should not be confused with the formal execution semantics to be presented below. Treating $\Pi$ as a tuple of messages, we denote its $i$ th message by $\Pi_{i}$. We denote the set of protocol users (participants) by $U=\left\{u_{1}, u_{2}, \ldots, u_{n}\right\}$, where any two of whom may initiate an instance of the protocol together, in a manner controlled by an adversary. The adversary is not himself a protocol user, but may dynamically subvert users during the execution. We model adversarial power as an oracle with which he is adaptively interacting, by making the following types of query:

- $\operatorname{corrupt}(i)$ : Corrupts user $u_{i}$. In response, the long-lived secret key of $u_{i}$ (and all other $u_{i}$ 's internal information) is given to the adversary.

- new-session $(i, j)$ : Causes $u_{i}$ and $u_{j}$ to start a new session, with $u_{i}$ as the initiator. The oracle assigns a unique number, sn, to their session and gives $s n$ to the adversary plus the first message that $u_{i}$ sends to $u_{j}$ in this session.

- $\operatorname{send}\left(s n, m_{1}, I\right)$ : Causes the oracle to send message $m_{1}$ to the initiator of session $s n$ and give $m_{2}$, the message that the user produces in response, to the adversary. Here $m_{2}$ may be a valid message, an error message $\perp$ (indicating $m_{1}$ was not of the right format), or a flag message * indicating that the user has received her last message, finishing her session.

$-\operatorname{send}\left(s n, m_{1}, R\right)$ : Similar to above, but $m_{1}$ is sent to the responder of $s n$.

We now give formal and computational semantics for protocols. In the formal setting, we denote the long-lived public key of $u_{i}$ by $k_{u_{i}}$, and for each session $s n$ that $u_{i}$ is a user of, we denote $u_{i}$ 's generated symmetric keys and nonces in $s n$, respectively, by $\mathbf{K}_{i, s n}^{s y m}=\left\{k_{i, s n, j}^{\text {sym }} \mid j \in \mathbb{N}\right\} \subseteq \mathcal{K}_{H}^{\text {privsym }}, \mathbf{X}_{i, s n}=\left\{x_{i, s n, j} \mid j \in\right.$ $\mathbb{N}\} \subseteq \mathcal{X}_{H}$. The adversary may use his own basic symbols to build new messages; we denote the adversary's symmetric keys and nonces, respectively, by $\mathbf{K}_{A}^{\text {sym }}=$ $\left\{k_{A, j}^{\text {sym }} \mid j \in \mathbb{N}\right\} \subseteq \mathcal{K}_{A}^{\text {privsym }}, \mathbf{X}_{A}=\left\{x_{A, j} \mid j \in \mathbb{N}\right\} \subseteq \mathcal{X}_{A}$. We let Exp basic be the union of all $\mathbf{X}_{A}, \mathbf{K}_{A}^{\text {sym }}, \mathbf{K}_{i, s n}^{\text {sym }}$ 's, $\mathbf{X}_{i, s n}$ 's.

The adversary initially knows only his own basic symbols and parties' IDs and public keys. If he corrupts $u_{i}$, he receives $k_{u_{i}}^{-1}$ as well as $\mathbf{K}_{i, s n}^{s y m} \cup \mathbf{X}_{i, s n}$, for every $s n$ that $u_{i}$ has engaged in. A protocol state is characterized by the following components:

$$
\begin{array}{lr}
f:\{I, R\} \times B S(\Pi) \times S N \rightarrow \operatorname{Exp}_{\text {basic }} \cup\{\perp\} & l:\{I, R\} \times S N \rightarrow \Pi_{i} \cup\{\sqrt{ }\} \\
h:\{I, R\} \times S N \rightarrow U & \text { corr-users } \subseteq\left\{u_{1}, \ldots, u_{n}\right\}
\end{array}
$$

Here $S N$ denotes the set of all session numbers, and, recall that, $U$ is the set of all protocol users. Function $f$ represents the symbolic values that the initiator and responder of each session of the protocol give to basic symbols in that session, and $\perp$ means that the party does not yet know the value of the corresponding basic message. Function $l$ denotes the index of the next message in the protocol that the initiator and responder of each session expect to receive, and $\sqrt{ }$ indicates that the party has finished her respective session. Finally function $h$ indicates what protocol users take the roles of "initiator" and "responder" in each session. 
We denote the initial state of the system by $F S_{0}$, where corr-users $=\emptyset$, and $l, f, h$ map all their inputs to null values. An execution of a formal adversary, $\mathcal{A}_{F}$, can be described as a sequence of queries $E\left(\mathcal{A}_{F}\right)=\left(q_{1}, q_{2}, \ldots\right)$, with corresponding replies $\left(r_{1}, r_{2}, \ldots\right)$. We then call $\mathcal{A}_{F}$ coinductively legitimate if $m \in$ closure $_{c}\left(r_{1}, r_{2}, \ldots, r_{i-1}\right)$ for all $i$ such that $q_{i}=\operatorname{send}(s n, m,\{I, R\})$. Under $\mathcal{A}_{F}$ 's execution, we denote the induced formal trace by $\mathcal{F} \mathcal{T}\left(\mathcal{A}_{F}\right)=\left(F S_{0}, F S_{1} \ldots\right)$, where state $F S_{i}$ is obtained from $F S_{i-1}$ as a result of query $q_{i}$.

Under the computational execution, elements of $B S(\Pi) \subseteq I d s \cup$ nonces $\cup$ pubkeys $\cup$ symkeys are replaced with random bitstrings, sampled w.r.t. a pair $\mathcal{E}_{p}=\left(\mathcal{E}_{\text {asy }}, \mathcal{E}_{\text {sym }}\right)$, with w.l.o.g. a shared security parameter $1^{\eta}$, and the coins tossed by both protocol users and the adversary during the protocol execution. Each (initially honest) $u_{i}$, before engaging in the protocol execution, samples her long-lived key pair, $\left(p k_{i}, s k_{i}\right) \leftarrow \operatorname{Gen}_{a s y}(\eta)$, and for each session $s n$ that $u_{i}$ participates in, $u_{i}$ uses a (polynomially-long) uniformly-selected random string $\mathcal{R}_{i, s n}$ to sample her nonces and symmetric keys in that session, where symmetric keys are sampled according to $G e n_{\text {sym }}$, and nonces chosen uniformly at random from a fixed nonce space, $N S=\{0,1\}^{\text {poly }(\eta)}$. The adversary, using random string $\mathcal{R}_{A}$, may choose his nonces and symmetric keys (to, e.g., replace those of corrupted parties, inject in messages on the network, etc.) in any arbitrary efficient manner; he may also initially corrupt a party and choose her public/private key pair in any arbitrary manner (not necessarily following Gen $_{a s y}$ ).

Letting $C_{\eta}=N S \cup \sup \left[\operatorname{Gen}_{\text {sym }}(\eta)\right] \cup \sup \left[\operatorname{Gen}_{\text {asy }}(\eta)\right]$, a computational state of the protocol is given by $(F, L, H, C o r r-U s e r s)$, where $L, H, C o r r-U s e r s$ are defined analogously to their formal counterparts, and $F$ is also defined similarly to $f$ by replacing $E x p_{b}$ with $C_{\eta}$. The adversary interacts with a computational oracle by issuing the four types of queries explained above, where the input/output of queries are probabilistic, depending on $\mathcal{R}_{A}$ and $\mathcal{R}_{H}$. (Here $\mathcal{R}_{H}$ is the concatenation of all random coins used by honest parties.) Among oracle queries, we only explain the effect of a corruption query (the others are fairly straightforward): if the adversary corrupts $u_{i}$, he is given $\left(p k_{i}, s k_{i}\right)$, and for every session $s n$ in which $u_{i}$ takes the role $X \in\{I, R\}$, the adversary is given $F(X, b s, s n)$, for every $b s \in B S(\Pi)$. Finally, under fixed $\mathcal{R}_{H}$ and $\mathcal{R}_{A}$, the induced computational trace is deterministic and denoted by $\mathcal{C} \mathcal{T}\left(\mathcal{A}, \mathcal{R}_{A}, \mathcal{R}_{H}, \Pi_{\mathcal{E}_{p}}\right)$.

Let $F T=\left\langle\left(f_{1}, l_{1}, h_{1}\right.\right.$, corr-users $\left.s_{1}\right),\left(f_{2}, h_{2}, l_{2}\right.$, corr-user $\left.\left.s_{2}\right), \ldots\right\rangle$ be a formal trace and let $\tau: \operatorname{Exp}_{\text {basic }} \rightarrow \mathcal{C}_{\eta}$ be a concrete mapping. We say a concrete trace

$$
C T=\left\langle\left(F_{1}, L_{1}, H_{1}, \text { Corr-Users }{ }_{1}\right),\left(F_{2}, L_{2}, H_{2}, \text { Corr-Users } s_{2}\right), \ldots\right\rangle
$$

is an encoding of FT under $\tau$, written $F T \prec_{\tau} C T$, if $l_{i}=L_{i}, h_{i}=H_{i}$, Corr-Users $=$ corr-users and $F_{i}=\tau f_{i}$, for all $i \geq 1$. We say $C T$ is the computational image of $F T$, written $F T \prec C T$, if there exists $\tau$ such that $F T \prec_{\tau} C T$.

We are now ready give the computational soundness definition.

Definition 1. A pair $\mathcal{E}_{p}=\left(\mathcal{E}_{\text {asy }}, \mathcal{E}_{\text {sym }}\right)$ provides a computationally-sound interpretation of symbolic encryption with respect to coinductive Dolev-Yao traces (shortly, provides soundness) if for all valid protocols $\Pi$, adversaries $\mathcal{A}_{c}$, we have

$$
\operatorname{Pr}_{\mathcal{R}_{A}, \mathcal{R}_{H}}\left[\exists\left\{\text { coind-legit } \mathcal{A}_{F}\right\}: \mathcal{F} \mathcal{T}\left(A_{F}\right) \prec \mathcal{C} \mathcal{T}\left(\mathcal{A}_{c}, \mathcal{R}_{A}, \mathcal{R}_{H}, \Pi_{\mathcal{E}_{p}}\right)\right] \geq 1-\operatorname{negl}(\eta)
$$




\section{Computational Realization of Coinductive Methods}

We describe a joint notion of security for asymmetric/symmetric encryption which provides soundness for coinductive symbolic traces. We then explore how this notion may be achieved under standard complexity-theoretic assumptions.

We begin with some motivation. Consider a single run of a protocol against a passive adversary, in which the whole sequence of exchanged messages is known a priori. We wish to formalize what it means for a piece of data (nonce or symmetric key) to remain secure in both the formal and computational settings. Under the formal approach, one would typically say the secrecy of a piece of data is retained if it cannot be deduced by applying Dolev-Yao rules. For a nonce $X$, for instance, if $X$ is not formally deducible, it means all occurrences of $X$ are encrypted under keys which cannot be obtained by a Dolev-Yao adversary. Thus, under the concrete instantiation, after the adversary has received the computational representations of the exchanged messages, the random nonce value underlying $X$ should still be as computationally random as a freshly-generated random nonce, provided the encryption scheme is sufficiently strong. However, for the case of symmetric keys the situation is quite different: even if a symmetric key is not Dolev-Yao-style deducible, the key may leak significant information when it comes to a concrete implementation. For instance, a symmetric-key value may lose its original randomness if used for encryption. (i.e. The adversary will be able to tell it apart from a fixed key, causing it to not be as "random" as a freshly generated key.) Thus the definition of secrecy for symmetric keys in the computational model turns out to be more delicate.

Our ultimate goal is to establish a close correspondence between coinductive Dolev-Yao adversaries and computational adversaries, by showing that a computational adversary essentially cannot do anything (in terms of mounting successful attacks) which cannot already be performed by a simple Dolev-Yao adversary. We capture the essence of active-attack scenario within a cryptographic game, played between an adversary and a challenger, in which the adversary is faced with a number of unknown keys (both asymmetric and symmetric) and nonces, generated by the challenger, and his goal is to infer "non-trivial" information from the challenger's secret data, by exploiting active attacks such as corrupting arbitrary keys of the challenger, getting her to encrypt messages which depend on her own secret data, and getting her to decrypt "permissible" ciphertexts. Our goal is to show that, under sufficiently strong security requirements, the computational adversary cannot learn non-trivial information from a piece of data (nonce or private key) that cannot already be obtained by a coinductive Dolev-Yao adversary. The key point in our security definition is to formalize the idea of "computational secrecy" for private keys. As it is probably clear from the above discussion, "requiring the adversary not be able to distinguish the private key (used in the game) from a freshly generated key" would not work. We formalize it in the following standard way: a private key retains its computational secrecy if the adversary is unable to distinguish between the encryptions of real/random messages under that key. We will be able to show that security in our game provides computational soundness. 
Our security notion is formalized via the following game which we call the coinductive, key-dependent indistinguishability game, or the CI game for short. Below, for $S=\left\{\left(s_{1}^{1}, s_{1}^{2}\right), \ldots\left(s_{n}^{1}, s_{n}^{2}\right)\right\}$, we define $S^{i} \triangleq\left\{s_{1}^{i}, \ldots, s_{n}^{i}\right\}$ for $i \in\{1,2\}$.

\subsection{Coinductive, Key-Dependent Indistinguishability (CI) Game}

Assume $\mathcal{E}_{a s y}=\left(G_{e n} n_{a s y}, E n c_{a s y}, D e c_{a s y}\right)$ and $\mathcal{E}_{\text {sym }}=\left(G_{e n} n_{\text {sym }}, E n c_{\text {sym }}\right.$, Dec $\left._{\text {sym }}\right)$ are asymmetric/symmetric encryption schemes whose joint security is to be defined, w.l.o.g., w.r.t. the shared security parameter $1^{\eta}$. The game is played between an adversary, $\mathcal{A}$, and a challenger, $\mathcal{B}$, and is parameterized over a publiclyknown, poly-bounded integer function $n(\eta)$ (we write $n$ for $n(\eta)$ ). Suppose $\tau_{B}(\cdot)$ and $\tau_{A}(\cdot)$ are (dynamically growing) mappings which give bitstring values to, respectively, the basic symbols of $\mathcal{B}$ and $\mathcal{A}$ (we will see shortly what those symbols are), and let $\tau$ be a mapping defined to be $\tau_{B}$ on the domain of $\mathcal{B}$ 's symbols and $\tau_{A}$ on $\mathcal{A}$ 's. Here $\tau_{A}$ is publicly known, while access to $\tau_{B}$ and $\tau$ is restricted to $\mathcal{B}$. The game proceeds in three phases: setup, interaction, and guessing.

In the setup phase, $\mathcal{B}$ first picks $b \leftarrow\{0,1\}$, generates $\left\{\left(p k_{i}, s k_{i}\right)\right\}_{1 \leq i \leq n} \leftarrow$ $\operatorname{Gen}_{\text {asy }}(\eta)$, symmetric keys $\left\{c k_{i}\right\}_{1 \leq i \leq n} \leftarrow \operatorname{Gen}_{\text {sym }}(\eta)$, and nonces $\left\{n c_{i}\right\}_{1 \leq i \leq n} \leftarrow$ $\{0,1\}^{q(\eta)}$ (for some poly $q$ ), makes $\left\{p k_{i}\right\}_{1 \leq i \leq n}$ public, and keeps the rest secret. We introduce $\left\{\left(k_{i}, k_{i}^{-1}\right)\right\}_{1 \leq i \leq n} \in \mathcal{K}_{H}^{p u b} \times \mathcal{K}_{H}^{\text {privasym }}$, and $\left\{k_{i}^{\text {sym }}\right\}_{1 \leq i \leq n} \in$ $\mathcal{K}_{H}^{\text {privsym }}$, and $\left\{x_{i}\right\}_{1 \leq i \leq n} \in \mathcal{X}_{H}$, and assign $\tau_{B}\left(k_{i}\right)=p k_{i}, \tau_{B}\left(k_{i}^{-1}\right)=s k_{i}$, $\tau_{B}\left(k_{i}^{s y m}\right)=c k_{i}$ and $\tau_{B}\left(x_{i}\right)=n c_{i}$, for $1 \leq i \leq n$. We initialize eval-exp $=\emptyset$. During the interaction phase, $\mathcal{A}$ may dynamically update $\tau_{A}$, mapping his newly-created basic symbols to arbitrary values. In the interaction phase $\mathcal{A}$ adaptively interacts with $\mathcal{B}$ by issuing queries of the following types:

1. Corruption: $\mathcal{A}$ may corrupt a $\mathcal{B}$ 's key by issuing $\operatorname{corrupt}(s)$, where $s \in$ $\left\{k_{1}^{-1}, \ldots, k_{n}^{-1}, k_{1}^{s y m}, \ldots, k_{n}^{s y m}\right\}$. In response $\mathcal{A}$ receives $\tau(s)$, and $(s, \tau(s))$ is added to eval-exp.

2. Encryption: $\mathcal{A}$ may issue a query encrypt $(e, x)$, where $x \in\left\{k_{1}, \ldots, k_{n}, k_{1}^{\text {sym }}\right.$ $\left., \ldots, k_{n}^{s y m}\right\}$, and $e$ may not have any $k_{i}^{-1}$ 's as a subexp. In response, $\mathcal{A}$ is given $c \leftarrow \llbracket\{e\}_{x} \rrbracket_{\tau}$ and $\left(\{e\}_{x}, c\right)$ is added to eval-exp. Here $e$ may contain both the challenger's and adversary's basic symbols.

3. Decryption: $\mathcal{A}$ may issue $\operatorname{decrypt}\left(c, s^{\prime}\right)$, where $s^{\prime} \in\left\{k_{1}^{-1}, \ldots, k_{n}^{-1}, k_{1}^{s y m}\right.$, $\left.\ldots, k_{n}^{s y m}\right\}$. In response $\mathcal{A}$ receives $\operatorname{Dec}_{a s y}\left(c, s k_{i}\right)$ if $s^{\prime}=k_{i}^{-1}$ and $\operatorname{Dec}_{s y m}\left(c, c k_{i}\right)$ if $s^{\prime}=k_{i}^{s y m}$, unless there exists $\left(\{e\}_{k_{p}}, c_{p}\right) \in$ eval-exp such that $\{e\}_{k_{p}}$ has a subexp $\left\{e^{\prime}\right\}_{s}$ (where $s^{\prime}=s^{-1}$ ) which in $\{e\}_{k_{p}}$ is encrypted only under keys whose decryption keys are in closure $\left(\right.$ eval-exp $\left.{ }^{1}\right)$, and that $c$ corresponds to the computational image of $\left\{e^{\prime}\right\}_{s}$ in $c_{p}$. In this case the answer is $\perp$.

After making a number of such queries, $\mathcal{A}$ proceeds to the final, guessing phase, in which he claims he is able to infer "non-trivial" information about irrecoverable secret data of $\mathcal{B}$. He does so by issuing a challenge query, which is either of the form challenge $(s)$, where $s \in\left\{x_{1}, \ldots, x_{n}\right\}$ (nonce challenge), or of the form challenge $(s, b s)$, where $b s \in\{0,1\}^{*}$ and $s \in\left\{k_{1}^{-1}, \ldots, k_{n}^{-1}, k_{1}^{s y m}, \ldots, k_{n}^{s y m}\right\}$ (secret key challenge.) The response to the query is decided as follows: if $s \in$ closure $_{c}\left(\right.$ eval-exp $\left.^{1}\right)$, then he is given $\perp$, otherwise: 
- if $b=0, \mathcal{A}$ is given $n c_{j}$ if $s=x_{j}, E n c_{a s y}\left(b s, p k_{j}\right)$ if $s=k_{j}^{-1}$, and otherwise $\operatorname{Enc}_{\text {sym }}\left(b s, c k_{j}\right)$ if $s=k_{j}^{s y m}$.

- if $b=1, \mathcal{A}$ is given $n c_{j}^{\prime} \leftarrow\{0,1\}^{q(\eta)}$ if $s=x_{j}, E n c_{a s y}\left(r, p k_{j}\right)$ if $s=k_{j}^{-1}$, and $\operatorname{Enc}_{\text {sym }}\left(r, c k_{j}\right)$ if $s=k_{j}^{\text {sym }}$, where $r \leftarrow\{0,1\}^{|b s|}$.

$\mathcal{A}$ finally outputs his guess for $b$. Denoting by $\mathcal{A}^{\mathrm{CI}_{b}^{\varepsilon_{p}}}$ the output of $\mathcal{A}$ when the secret bit is $b$, his CI-advantage is (below $\mathcal{E}_{p}$ refers to the pair of schemes):

$$
A d v_{\mathcal{E}_{p}, \mathcal{A}}^{\mathrm{CI}}(\eta)=\left|\operatorname{Pr}\left[\mathcal{A}^{\mathrm{CI}_{b}^{\mathcal{E}_{p}}}(\eta)=1 \mid b=0\right]-\operatorname{Pr}\left[\mathcal{A}^{\mathrm{CI}_{b}^{\mathcal{E}_{p}}}(\eta)=1 \mid b=1\right]\right| .
$$

Definition 2. A pair of $\mathcal{E}_{p}=\left(\mathcal{E}_{\text {sym }}, \mathcal{E}_{\text {asy }}\right)$ provides joint security under the $C I$ game (shortly, is CI-secure) if for every $\mathcal{A}, A d v_{\mathcal{E}_{p}, \mathcal{A}}^{I I}(\eta)$ is negligible.

We now explain the restrictions on challenge and decryption queries. For our discussion, assume that $\mathcal{E}=(G e n, E n c, D e c)$ is a symmetric encryption scheme wherein $E n c(c k, c k)$ leads to computation of $c k$. (This could happen although $\mathcal{E}$ is secure in any standard sense.) In the absence of the condition for challenge queries, $\mathcal{A}$ could simply win the game by doing the following: make two queries encrypt $\left(k_{1}^{\text {sym }}, k_{1}^{\text {sym }}\right)$ and encrypt $\left(k_{2}^{\text {sym }}, k_{1}^{\text {sym }}\right)$ to receive, respectively, $c_{1}$ and $c_{2}$, and then issue the challenge query challenge $\left(k_{2}^{s y m}, 0^{n}\right) ; \mathcal{A}$ may now obtain $\tau\left(k_{1}^{\text {sym }}\right)$ from $c_{1}$ and $\tau\left(k_{2}^{\text {sym }}\right)$ from $c_{2}$, trivially winning the game. Also in the absence of the condition for decryption queries, $\mathcal{A}$ could simply win as follows: (1) make two queries encrypt $\left(k_{1}^{\text {sym }}, k_{1}^{\text {sym }}\right)$ and encrypt $\left(\left\{k_{2}^{\text {sym }}\right\}_{k_{3}^{\text {sym }}}, k_{1}^{\text {sym }}\right)$ to receive, respectively, $c_{1}$ and $c_{2}$, (2) after computing $\tau\left(k_{1}^{\text {sym }}\right)$ from $c_{1}$, issue the decryption query decrypt $\left(c_{3}, k_{3}^{\text {sym }}\right)$, where $c_{3}=\operatorname{Dec}\left(c_{2}, \tau\left(k_{1}^{\text {sym }}\right)\right)$, and (3) after obtaining $\tau\left(k_{2}^{\text {sym }}\right)$ issue the challenge query challenge $\left(k_{2}^{\text {sym }}, 0^{n}\right)$, trivially winning the game. Finally we remark that the recent results of [20] show that there exists an IND-CCA2-secure symmetric encryption scheme such that ciphertexts $\operatorname{Enc}\left(c k_{1}, c k_{2}\right), \ldots, E n c\left(c k_{n-1}, c k_{n}\right), E n c\left(c k_{n}, c k_{1}\right)$, for randomly-generated $c k_{i}$ 's, lead to revelation of all $c k_{1}, \ldots, c k_{n}$ (a weaker case than $k$-circular security). Therefore, the above attack methods extend to longer key cycles.

Note, $\mathcal{A}$ may use an encrypt query to obtain the encryption of any bitstring. For example, to encrypt $m$ under $c k_{i}$, he may introduce a new basic symbol $x_{\mathcal{A}}$, set $\tau_{\mathcal{A}}\left(x_{\mathcal{A}}\right)=m$, and then issue encrypt $\left(x_{\mathcal{A}}, k_{i}^{\text {sym }}\right)$. Also it is possible to define and extend results we present about CI security to a (seemingly) stronger notion in which $\mathcal{A}$ is allowed to make multiple challenge queries, possibly making them interleave with the other types of queries. Right now for applications that we consider, CI security suffices. CI security may be thought of as a variant of KDM security with the underlying function family consisting of any arbitrary composition of constant, projection, pairing and encryption functions. However, since we aim to prove generic implication results from standard security definitions, we have to restrict the set of keys for which we want to prove computational secrecy (i.e. those which remain coinductively irrecoverable). This differs from KDM security in which one wants to prove computational secrecy for all keys, regardless of what encryption queries were made. Finally we stress that a key $\mathcal{A}$ challenges in the guessing phase may have previously occurred in key cycles. 
CI security is still insufficient for providing soundness as it does not provide integrity of ciphertexts. To account for this, we strengthen it to also provide ciphertext integrity and call the new notion authenticated CI (or ACI) security. We say $\left(\mathcal{E}_{\text {asy }}, \mathcal{E}_{\text {sym }}\right)$ is $A C I$ secure if it is CI secure and further any $\mathcal{A}$ has a negligible chance of winning in the ACI game defined as follows: the setup and interaction phases proceed exactly as in the CI game, while in the guessing phase, $\mathcal{A}$ outputs $(c, i)$ and wins if: (1) $\operatorname{Dec}_{\text {sym }}\left(c, c k_{i}\right) \neq \perp$, and (2) there does not exist $\left(\{e\}_{k_{j}}, c^{\prime}\right) \in$ eval-exp such that $\{e\}_{k_{j}}$ has a subexp $\left\{e^{\prime}\right\}_{k_{i}}$ encrypted in $\{e\}_{k_{j}}$ only under keys whose decryption keys are in closure (eval-exp $^{1}$ ), and that $c$ is the corresponding image of $\left\{e^{\prime}\right\}_{k_{i}}$ in $c^{\prime}$.

As a step toward proving soundness with respect to ACI security, we formulate a new notion which characterizes security requirements capturing the basic Dolev-Yao assumptions made in protocol analysis, and prove that it provides soundness. Our notion, which we call coinductive, key-dependent non-malleability (shortly $C N M$ ) notion, is a generalization of the Dolev-Yao non-malleability notion of [28, which was defined for the passive setting.

\subsection{Coinductive, Key-Dependent Non-Malleability (CNM) Game}

The game is parameterized, again, over $\mathcal{E}_{p}=\left(\mathcal{E}_{a s y}, \mathcal{E}_{s y m}\right)$, a shared security parameter $\eta$, and a computational mapping $\tau$, and runs in three phases with the setup and interaction phases as in the CI game (except that no $b$ is sampled). However, in the guessing phase, $\mathcal{A}$ claims he is able to construct the computational image of an expression which is not coinductively constructible from eval-exp ${ }^{1}$. To this end, he outputs $(e, E)$, where $e \in \operatorname{Exp}$ (containing, possibly, both the adversary's and challenger's symbols) and $E \in\{0,1\}^{*}$. The output of the game is 1 , written as $\operatorname{CNM}_{\mathcal{E}_{p}, \eta}(\mathcal{A})=1$, if the following two conditions hold:

1. $e \notin$ closure $_{c}\left(\right.$ eval-exp $\left.^{1}\right)$; and

2. $E$ is a possible mapping of $e$ under $\tau$ and $\mathcal{E}_{p}$; namely, $E \in \llbracket e \rrbracket_{\tau}^{\mathcal{E}_{p}}$. Note condition (2) is efficiently verifiable given access to $\tau$. We define

$$
A d v_{\mathcal{E}_{p}, \eta}^{\mathrm{CNM}}(\mathcal{A})=\operatorname{Pr}\left[\mathrm{CNM}_{\mathcal{E}_{p}, \eta}(\mathcal{A})=1\right] .
$$

Definition 3. A pair $\mathcal{E}_{p}=\left(\mathcal{E}_{\text {asy }}, \mathcal{E}_{\text {sym }}\right)$ provides security under the CNM game (shortly, is $C N M$-secure) if for every adversary $\mathcal{A}, \operatorname{Adv}_{\mathcal{E}_{p}, \eta}^{C N M}(\mathcal{A})$ is negligible.

Theorem 1. 1. CNM security $\Rightarrow$ soundness

2. $A C I$ security $\Rightarrow C N M$ security.

Proof (Outline): For (2) if $\mathcal{A}^{c n m}$ is able to output a CNM-valid $(e, E)$, then $e \notin T$, where $T=$ closure $_{c}\left(\right.$ eval-exp $\left.^{1}\right)$ implies $e$ has a subexp $s$ such that $s$ is either a nonce/private key, or $s=\{\cdot\}_{k_{j}^{s y m}}$, and that any subexp of $e$ which contains $s$ is not in $T$. This implies the underlying value of $s$ is recoverable from $E$ (with the aid of the decryption oracle) through successive decryptions down along the path leading to $s$, which will then enable an attack either against CI 
security or ciphertext integrity depending on the type of $s$. The proof for (1) also follows using ideas similar to those of [34. We give a full proof in [27].

For an adversary $\mathcal{A}$ in either of the above games, we define a labeled key graph, $G(\mathcal{A})=\left(V_{\mathcal{A}}, E_{\mathcal{A}}\right)$, as follows: $V_{\mathcal{A}}=\left\{v_{1}^{a s y}, \ldots, v_{n}^{a s y}, v_{1}^{s y m}, \ldots, v_{n}^{s y m}\right\}$, and $v_{i}^{x} \stackrel{a}{\rightarrow}$ $v_{j}^{y} \in E_{\mathcal{A}}$, for $x, y \in\{a s y$, sym $\}$ and $a \in \mathbb{N}$, if $k_{i}^{x}$ encrypts the ath occurrence of $\left(k_{j}^{y}\right)^{-1}$ in the sequence of $\mathcal{A}$ 's encryption queries. Here ath occurrence refers to an increasing numbering given to each decryption key as it appears in the sequence; for example, if $e_{1}=\left\{k_{1}^{s y m}, k_{p}^{s y m}\right\}_{k_{3}}, k_{p}^{s y m}$ and $e_{2}=k_{2}^{s y m},\left\{k_{p}^{s y m}\right\}_{k_{4}}$ and the first two encryption queries are encrypt $\left(e_{1}, k_{3}\right)$ and encrypt $\left(e_{2}, k_{5}\right)$; the set of keys that encrypt the 3 rd occurrence of $k_{p}^{s y m}$ is $\left\{k_{4}, k_{5}\right\}$. We call $v_{i}^{x}$ coinductively irrecoverable (irrecoverable for short) if $k_{i}^{x-1} \notin$ closure $_{c}\left(\right.$ eval-exp $\left.^{1}\right)$, and we refer to the induced subgraph on irrecoverable nodes as the hidden subgraph. The diameter of a graph is the length of the longest path in the graph. We define $\operatorname{indeg}\left(v_{i}^{x}\right)$ to be the maximum $a$ for which we have an incoming edge with label $a$ to $v_{i}^{x}$; this specifies the number of times $k_{i}^{x-1}$ occurs in $\mathcal{A}$ 's encryption queries. Note, $\operatorname{indeg}\left(v_{i}^{a s y}\right)=0$, for every $1 \leq i \leq n$, and also both $G(\mathcal{A})$ and indeg $\left(v_{i}\right)$ are random variables depending on the coins tossed during the game.

If all encryption queries were of the form encrypt $\left(k_{i}^{s y m}, k_{j}^{x}\right)$ (i.e. single encryptions) without cycle creation, then all nodes from which there was a path to an irrecoverable node would also be irrecoverable. However, in the case of nested encryptions with key cycles, the above appealing property no longer holds; namely, an irrecoverable node may occur in certain key cycles, and may have edges from nodes which are recoverable. For example, assuming $e_{1}=\left\{k_{1}^{\text {sym }}\right\}_{k_{2}^{\text {sym }}}$ and $e_{2}=\left\{k_{3}^{s y m}\right\}_{k_{4}^{s y m}}$, if $\mathcal{A}$ makes queries encrypt $\left(e_{1}, k_{5}^{s y m}\right)$, encrypt $\left(k_{2}^{s y m}, k_{1}^{s y m}\right)$, encrypt $\left(e_{2}, k_{6}^{s y m}\right)$, and corrupt $\left(k_{4}^{s y m}\right)$, all keys except $k_{4}^{s y m}$ remain irrecoverable, and there exists, for instance, edges in both directions between $v_{1}^{s y m}$ and $v_{2}^{\text {sym }}$ in $G(\mathcal{A})$.

However, in the case of cyclic nested encryption, we will base our hybrid arguments on a provable property, which we call coinductive continuability, of irrecoverable nodes. In $G(\mathcal{A})$, we say $v_{y_{1}}^{x} \stackrel{a_{2}}{\longrightarrow} v_{y_{2}}^{\text {sym }} \stackrel{a_{3}}{\longrightarrow} \ldots \stackrel{a_{p}}{\longrightarrow} v_{y_{p}}^{\text {sym }}$, for $x \in$ $\{$ sym,asy\}, is a coinductively continuable path if the following conditions hold: (below for better clarity we drop the superscripts $x$ and sym.)

1. Path validity: For all $2 \leq i \leq p, v_{y_{i-1}} \rightarrow^{a_{i}} v_{y_{i}} \in E_{\mathcal{A}}$, and if $1 \leq w<h \leq p$ then $v_{y_{w}} \neq v_{y_{h}}$,

2. For all $s \in\left\{k_{y_{1}}^{x-1}, k_{y_{2}}^{s y m}, \ldots, k_{y_{p}}^{\text {sym }}\right\}$ it holds $s \notin$ closure $_{c}\left(\right.$ eval-exp $\left.^{1}\right)$, and

3. either $\operatorname{indeg}\left(v_{y_{1}}\right)=0$ or for every $1 \leq a_{1} \leq \operatorname{indeg}\left(v_{y_{1}}\right)$ there exists $v_{i}^{w}$, with $w \in\{$ asy, sym $\}$, such that $v_{i}^{w} \stackrel{a_{1}}{\longrightarrow} v_{y_{1}} \stackrel{a_{2}}{\longrightarrow} \ldots \stackrel{a_{p}}{\longrightarrow} v_{y_{p}}$ is a coinductively continuable path.

We call $v_{i}^{x}$ coinductively continuable if its associated path of length zero is so.

Lemma 1. At any point, any irrecoverable node is coinductively continuable.

Proof (Outline): We prove this by an induction over the length of the longest path ending in the irrecoverable node. A full proof is given in [27]. 
Definition 4. We say that $\mathcal{E}_{p}=\left(\mathcal{E}_{\text {asy }}, \mathcal{E}_{\text {sym }}\right)$ provides l-CI security if $A d v_{\mathcal{E},{ }_{\mathcal{A}}(\eta)}^{C I}(\eta)$ is negligible for every $\mathcal{A}$ for whom the diameter of the hidden subgraph of $G(\mathcal{A})$ is always at most $l$. We say $\mathcal{E}_{p}$ provides $l$-ACI security if it is l-CI secure and any $\mathcal{A}$ (under the ACI game) for which the diameter of the resulting hidden subgraph is always at most $l$ has a negligible advantage.

Theorem 2. If $\mathcal{E}_{\text {asy }}$ and $\mathcal{E}_{\text {sym }}$ are both IND-CCA2 secure, then $\left(\mathcal{E}_{\text {asy }}, \mathcal{E}_{\text {sym }}\right)$ provides l-CI security, for every constant $l$.

Proof (Outline): The central idea is to guess a "random", coinductively continuable path, with some associated parameters, which ends in the challenge key, give "fake" values to certain private keys occurring as plaintexts, and prove the adversary's advantage under this replying strategy is negligibly different from that under the standard game. A full proof is given in [27].

Theorem 3. If $\mathcal{E}_{\text {asy }}$ provides IND-CCA2 security, and $\mathcal{E}_{\text {sym }}$ provides both IND$C C A 2$ and INT-CTXT security, then $\left(\mathcal{E}_{\text {asy }}, \mathcal{E}_{\text {sym }}\right)$ provides l-ACI security, for every constant $l$.

Proof (Outline): We first show if $\mathcal{A}_{a c i}$ is able to output an ACI-valid $(c, i)$, then in a world, $W_{i}$, in which occurrences of $k_{i}^{s y m}$ as a plaintext and its occurrences as an encryption key are given two independent values, $\mathcal{A}_{a c i}$ should have "the same" probability of producing a valid $(c, i)$, or otherwise a CI-attack can be made. Next, we show if under $W_{i}$ an adversary $\mathcal{A}$ is able to produce an ACI-valid $(c, i)$ and $c$ is already a plaintext of a ciphertext obtained under an encryption query (e.g. $\mathcal{A}$ has called encrypt $\left(\left\{x_{1}\right\}_{k_{i}^{\text {sym }}}, k_{2}^{\text {sym }}\right)$ to obtain $c_{2}, k_{2}^{\text {sym }}$ remains coinductively irrecoverably, and $\left.c=\operatorname{Dec}_{\text {sym }}\left(c_{2}, c k_{2}\right)\right)$, then a CI attack follows, and otherwise an INT-CTXT attack follows. A full proof is given in [27].

\section{Conclusion}

We investigated soundness of coinductive methods in a protocol model allowing arbitrary composition of symmetric/asymmetric encryption, as well as unrestricted transmission of secret keys. In such situations, an active adversary may selectively influence the encryption ordering between deployed keys, dynamically compromise them (naturally or under his corruption power), and potentially obtain encryption cycles. Any weakness in the underlying encryption schemes in the face of such an adversary may lead to insecure instantiations of protocols. Most previous work on computationally sound symbolic analysis of protocols either does not allow symmetric encryption, or imposes restrictions aimed at avoiding the above possibilities. Our soundness theorem, founded on coinduction, does not assume any such restrictions, while providing strong computational security guarantees against adaptive corruptions. Our results, however, rely on a property of protocols we call boundedness (formalized in [27]), which requires that no symbolic execution of the underlying protocol produce a coinductivelyirrecoverable encryption chain of nonconstant length. We observe that almost 
all protocols from [21] (when run in isolation) admit (at most) 2-boundedness. (All of them are bounded.) In [27, we provide statements on how one can reason about boundedness of a protocol, and whether the boundedness property is retained when two (individually bounded) protocols are run concurrently.

While the main focus of this paper is on trace-based security, we believe similar results can also be proved for key-exchange (KE) security tasks. A central security requirement for key exchange is the secrecy condition, requiring a secret key exchanged by a KE protocol be indistinguishable from a freshly generated key. Our CI game is rich enough to encompass common features of a KE attack model, including adaptive corruptions of users and session keys, while guaranteeing that (under stated complexity assumptions) coinducitve symbolic secrecy under the game implies computational secrecy (real-or-random indistinguishability in the case of nonces and key usability [23] in the case of secret keys).

For simplicity we have assumed if a user is corrupted, the adversary obtains only her long-lived key and her past generated secret keys/nonces, but not her past random coins. In [27] we give partial results about this more general case.

As briefly explained in the introduction, current results about KDM security do not seem sufficient for (unrestricted) secure realizations of protocols with inductive, symbolic security proofs. It would be interesting to extend (and realize) KDM security definitions to support adaptive corruptions. As pointed out earlier, defining the extension in an entirely left-or-right indistinguishability sense, as in 5], would entail inherent limitations; for example, if a left-or-right encryption query is made under $c k$, then $c k$ cannot be corrupted afterward.

Finally it would be interesting to improve the bounds imposed by our soundness theorem (and those of [36]), and investigate its extensions to more general cryptographic frameworks supporting compositional reasoning [6]19].

\section{References}

1. Abadi, M., Rogaway, P.: Reconciling Two Views of Cryptography (the computational soundness of formal encryption). In: Watanabe, O., Hagiya, M., Ito, T., van Leeuwen, J., Mosses, P.D. (eds.) TCS 2000. LNCS, vol. 1872, pp. 3-22. Springer, Heidelberg (2000)

2. Acar, T., Belenkiy, M., Bellare, M., Cash, D.: Cryptographic Agility and Its Relation to Circular Encryption. In: Gilbert, H. (ed.) EUROCRYPT 2010. LNCS, vol. 6110, pp. 403-422. Springer, Heidelberg (2010)

3. Backes, M., Dürmuth, M., Unruh, D.: OAEP Is Secure under Key-Dependent Messages. In: Pieprzyk, J. (ed.) ASIACRYPT 2008. LNCS, vol. 5350, pp. 506-523. Springer, Heidelberg (2008)

4. Backes, M., Pfitzmann, B.: Symmetric encryption in a simulatable dolev-yao style cryptographic library. In: CSFW, pp. 204-218. IEEE Computer Society (2004)

5. Backes, M., Pfitzmann, B., Scedrov, A.: Key-dependent message security under active attacks - brsim/uc-soundness of symbolic encryption with key cycles. In: CSF, pp. 112-124. IEEE Computer Society (2007)

6. Backes, M., Pfitzmann, B., Waidner, M.: A composable cryptographic library with nested operations. In: Jajodia, S., Atluri, V., Jaeger, T. (eds.) ACM Conference on Computer and Communications Security, pp. 220-230. ACM (2003) 
7. Bana, G., Comon-Lundh, H.: Towards Unconditional Soundness: Computationally Complete Symbolic Attacker. In: Degano, P., Guttman, J.D. (eds.) POST 2012. LNCS, vol. 7215, pp. 189-208. Springer, Heidelberg (2012)

8. Barthe, G., Daubignard, M., Kapron, B.M., Lakhnech, Y.: Computational indistinguishability logic. In: Al-Shaer, E., Keromytis, A.D., Shmatikov, V. (eds.) ACM Conference on Computer and Communications Security, pp. 375-386. ACM (2010)

9. Bellare, M., Boldyreva, A., Micali, S.: Public-Key Encryption in a Multi-user Setting: Security Proofs and Improvements. In: Preneel, B. (ed.) EUROCRYPT 2000. LNCS, vol. 1807, pp. 259-274. Springer, Heidelberg (2000)

10. Bellare, M., Desai, A., Pointcheval, D., Rogaway, P.: Relations among Notions of Security for Public-Key Encryption Schemes. In: Krawczyk, H. (ed.) CRYPTO 1998. LNCS, vol. 1462, pp. 26-45. Springer, Heidelberg (1998)

11. Bellare, M., Namprempre, C.: Authenticated encryption: Relations among notions and analysis of the generic composition paradigm. J. Cryptology 21(4), 469-491 (2008)

12. Bellare, M., Rogaway, P.: Entity Authentication and Key Distribution. In: Stinson, D.R. (ed.) CRYPTO 1993. LNCS, vol. 773, pp. 232-249. Springer, Heidelberg (1994)

13. Bellare, M., Rogaway, P.: Provably secure session key distribution: the three party case. In: Leighton, F.T., Borodin, A. (eds.) STOC, pp. 57-66. ACM (1995)

14. Boneh, D., Halevi, S., Hamburg, M., Ostrovsky, R.: Circular-Secure Encryption from Decision Diffie-Hellman. In: Wagner, D. (ed.) CRYPTO 2008. LNCS, vol. 5157, pp. 108-125. Springer, Heidelberg (2008)

15. Burrows, M., Abadi, M., Needham, R.: A logic of authentication. ACM Trans. Comput. Syst. 8, 18-36 (1990)

16. Camenisch, J., Chandran, N., Shoup, V.: A Public Key Encryption Scheme Secure against Key Dependent Chosen Plaintext and Adaptive Chosen Ciphertext Attacks. In: Joux, A. (ed.) EUROCRYPT 2009. LNCS, vol. 5479, pp. 351-368. Springer, Heidelberg (2009)

17. Canetti, R.: Universally composable security: A new paradigm for cryptographic protocols. In: FOCS, pp. 136-145. IEEE Computer Society (2001)

18. Canetti, R., Feige, U., Goldreich, O., Naor, M.: Adaptively secure multi-party computation. In: Miller, G.L. (ed.) STOC, pp. 639-648. ACM (1996)

19. Canetti, R., Herzog, J.: Universally Composable Symbolic Analysis of Mutual Authentication and Key-Exchange Protocols. In: Halevi, S., Rabin, T. (eds.) TCC 2006. LNCS, vol. 3876, pp. 380-403. Springer, Heidelberg (2006)

20. Cash, D., Green, M., Hohenberger, S.: New Definitions and Separations for Circular Security. In: Fischlin, M., Buchmann, J., Manulis, M. (eds.) PKC 2012. LNCS, vol. 7293, pp. 540-557. Springer, Heidelberg (2012)

21. Clark, J., Jacob, J.: A survey of authentication protocol literature. Technical report (1997)

22. Comon-Lundh, H., Cortier, V.: Computational soundness of observational equivalence. In: Ning, P., Syverson, P.F., Jha, S. (eds.) ACM Conference on Computer and Communications Security, pp. 109-118. ACM (2008)

23. Datta, A., Derek, A., Mitchell, J.C., Warinschi, B.: Computationally sound compositional logic for key exchange protocols. In: CSFW, pp. 321-334. IEEE Computer Society (2006)

24. Dolev, D., Yao, A.C.: On the security of public key protocols. In: Annual IEEE Symposium on Foundations of Computer Science, pp. 350-357 (1981)

25. Gilbert, H. (ed.): EUROCRYPT 2010. LNCS, vol. 6110. Springer, Heidelberg (2010) 
26. Goldwasser, S., Micali, S.: Probabilistic encryption. J. Comput. Syst. Sci. 28(2), 270-299 (1984)

27. Hajiabadi, M., Kapron, B.M.: Computational soundness of coinductive symbolic security under active attacks. IACR Cryptology ePrint Archive, 2012:560 (2012)

28. Herzog, J.: A computational interpretation of dolev-yao adversaries. Theor. Comput. Sci. 340(1), 57-81 (2005)

29. Impagliazzo, R., Kapron, B.M.: Logics for reasoning about cryptographic constructions. J. Comput. Syst. Sci. 72(2), 286-320 (2006)

30. Küsters, R., Tuengerthal, M.: Computational soundness for key exchange protocols with symmetric encryption. In: Al-Shaer, E., Jha, S., Keromytis, A.D. (eds.) ACM Conference on Computer and Communications Security, pp. 91-100. ACM (2009)

31. Lincoln, P., Mitchell, J.C., Mitchell, M., Scedrov, A.: A probabilistic poly-time framework for protocol analysis. In: Gong, L., Reiter, M.K. (eds.) ACM Conference on Computer and Communications Security, pp. 112-121. ACM (1998)

32. Micciancio, D.: Computational Soundness, Co-induction, and Encryption Cycles. In: Gilbert, H. (ed.) EUROCRYPT 2010. LNCS, vol. 6110, pp. 362-380. Springer, Heidelberg (2010)

33. Micciancio, D., Panjwani, S.: Adaptive Security of Symbolic Encryption. In: Kilian, J. (ed.) TCC 2005. LNCS, vol. 3378, pp. 169-187. Springer, Heidelberg (2005)

34. Micciancio, D., Warinschi, B.: Soundness of Formal Encryption in the Presence of Active Adversaries. In: Naor, M. (ed.) TCC 2004. LNCS, vol. 2951, pp. 133-151. Springer, Heidelberg (2004)

35. Mitchell, J.C., Ramanathan, A., Scedrov, A., Teague, V.: A probabilistic polynomial-time process calculus for the analysis of cryptographic protocols. Theor. Comput. Sci. 353(1-3), 118-164 (2006)

36. Panjwani, S.: Tackling Adaptive Corruptions in Multicast Encryption Protocols. In: Vadhan, S.P. (ed.) TCC 2007. LNCS, vol. 4392, pp. 21-40. Springer, Heidelberg (2007)

37. Pfitzmann, B., Waidner, M.: A model for asynchronous reactive systems and its application to secure message transmission. In: IEEE Symposium on Security and Privacy, pp. 184-200 (2001)

38. Rackoff, C., Simon, D.R.: Non-interactive Zero-Knowledge Proof of Knowledge and Chosen Ciphertext Attack. In: Feigenbaum, J. (ed.) CRYPTO 1991. LNCS, vol. 576, pp. 433-444. Springer, Heidelberg (1992) 